\title{
The Effect of Acetophenone and alpha-Methylstyrene on the Space Charge Properties of Low Density Polyethylene
}

\author{
N. Hussin and G. Chen \\ School of Electronics and Computer Science \\ University of Southampton, SO16 1BJ, UK \\ gc@ecs.soton.ac.uk
}

\begin{abstract}
In this report the pulsed elcetroacoustic (PEA) technique was employed to examine the space charge pattern in acetophenone and alpha-methylstyrene soaked low-density polyethylene (LDPE) samples. The samples were stressed at three different voltages; $5 \mathrm{kV}, 8 \mathrm{kV}$ and $10 \mathrm{kV}$, and the charge patterns in the sample bulk were compared with that obtained from the clean LDPE. These chemicals are observed to assist the transportation of the charges in the sample bulk. Each of these chemicals is in favour of different charge polarity. It seems that acetophenone assists the transportation of negative charges meanwhile the transportation of positive charges is assisted by the alphamethylstyrene. It is proposed that the chemicals provide shallow traps that aid the movement of the charges.
\end{abstract}

\section{INTRODUCTION}

In 1955, crosslinked polyethylene (XLPE) was first patented and with the crosslinking technique, the thermal stability of PE was improved [1]. XLPE is now been used widely in high voltage insulation system. Compare to LDPE, XLPE has slightly higher dielectric loss, last longer and less moisture sensitive [2]. However, despite of its ability to withstand high temperature, easy accumulation of space charge in XLPE become the main concern in employing this material in insulation system [3]. Dicumyl Peroxide (DCP) which is widely used as the crosslinking agent creates volatile crosslinking by-products such as acetophenone, cumyl alcohol, and $\alpha$-methylstyrene which also affect the insulation properties. To be able to develop insulating materials for insulating cables, it is essential to have a firm understanding of the influence of those additives to the electrical performance of insulation system. For this reason, the paper reports on the influence of acetophenone and alpha-methylstyrene on space charge characteristics in LDPE using the PEA technique.

\section{EXPERIMENTAL DETAILS}

All samples that are used in this project are supplied by GoodFellow. The samples with a thickness of $180 \mu \mathrm{m}$ were cut into small round film with a diameter of $3.6 \mathrm{~cm}$. In this project, LDPE was chosen over XLPE to eliminate any possibility of having more than one byproduct in the sample. Although XLPE can be degassed to remove all byproducts in the sample, but there is a possibility to have some byproducts left in the insulator [4].

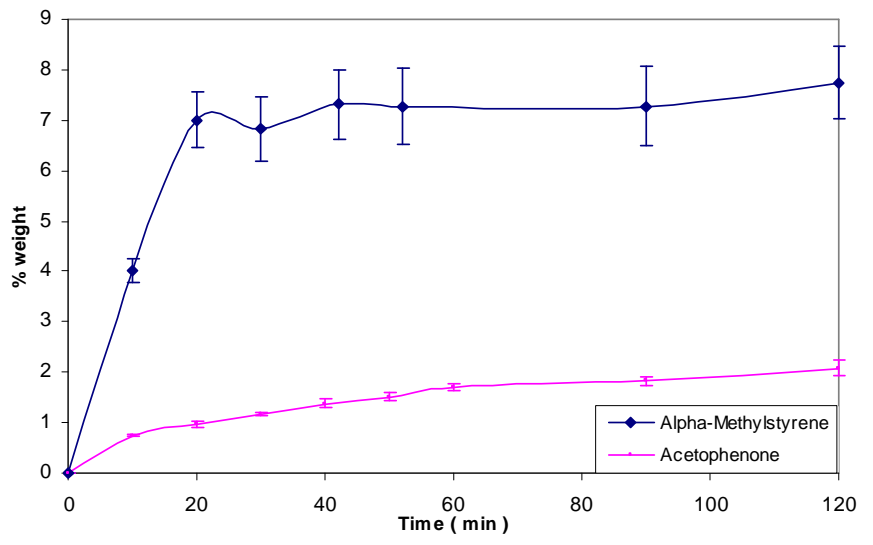

Figure 1: The soaking rate of acetophenone and $\alpha$-methylstyrene in LDPE

The samples are soaked into $\alpha$-methylstyrene and acetophenone for 2 hours at room temperature. The absorption rate can be observed by percentage weight increase that is presented in Figure 1. The percentage weight increases dramatically during the first 20 minutes, and then it gradually increases by time. After one hour, the percentage of $\alpha$ methylstyrene is about 4 times larger than acetophenone due to low permeation velocity into LDPE. This result shows that by soaking LDPE sample into the chemicals for 2 hours, the amount of them in the sample is sufficient enough for electrical test to be conducted on it.

In the space charge measurement, a pulse voltage of $600 \mathrm{~V}$ with duration of $5 \mathrm{~ns}$ is applied to the sample to generate an acoustic signal wave. Both soaked samples and the clean LDPE have been stressed at three different dc voltages; $5 \mathrm{kV}$, $8 \mathrm{kV}$ and $10 \mathrm{kV}$. Readings were taken for every 10 minutes for one hour and after that the charge decay pattern was monitored for another one hour. Before the samples were stressed at the specified voltage, $2 \mathrm{kV}$ dc voltage was applied to the sample for calibration purpose which had been discussed in [5].

\section{RESULTS AND DISCUSSION}

\section{Volts off results}

Before we could analyse the accumulation of space charge in the soaked sample, it is important to study the charge 

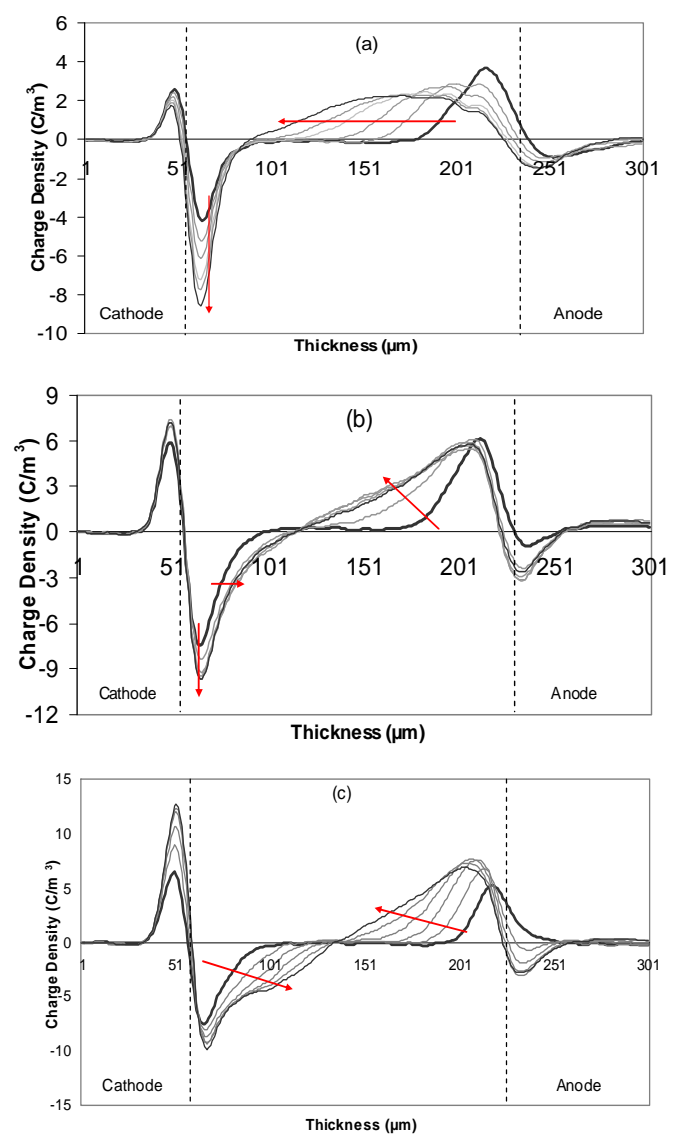

Figure 2: Charge dynamics in the clean LDPE; a) stressed at $5 \mathrm{kV}, \mathrm{b}$ ) stressed at $8 \mathrm{kV}, \mathrm{c}$ ) stressed at $10 \mathrm{kV}$, during volts off condition.

behaviours in the clean LDPE so that the analysis of the space charge accumulation will not be deluded. To reveal charge formation and movement more clearly, the results from the measurement of volts off are used in the present paper. The charge build up in the clean LDPE can be clearly seen in Figure 2. As ageing time increases, the charges of both polarities gradually increase in the sample that is stressed at $5 \mathrm{kV}$. When the voltage increases to $8 \mathrm{kV}$ and then $10 \mathrm{kV}$, more negative charges injected from the bottom electrode causing negative charge residential near the bottom electrode. In the sample stressed at $10 \mathrm{kV}$, the total negative and positive charge in the bulk is in balance. It is clear that the meeting point of positive and negative charges moves towards the middle of the sample with the increasing applied voltage.

For acetophenone soaked LDPE sample, the space charge results at the three voltages are illustrated in Figure 3. The amount of charge present in the sample generally increases with the applied voltage. This time, the domination of negative charges is even more obvious. In the vicinity of the anode, the amount of positive charge decreases with time indicating either the positive charges migrating towards the cathode, or the amount of negative charges overcoming the numbers of positive charges in that particular region. It can be perceived that as the stressing time increases, more negative charges migrate into the bulk, moving towards the anode. This
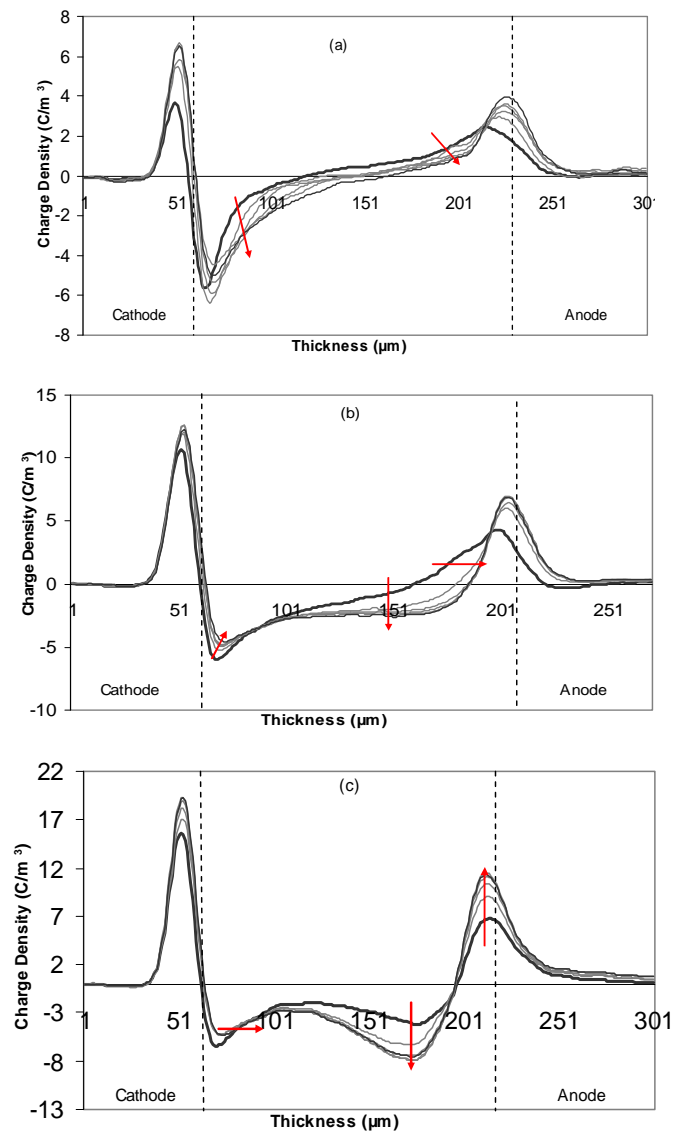

Figure 3: Charge dynamics in acetophenone soaked LDPE; a) stressed at $5 \mathrm{kV}$, b) stressed at $8 \mathrm{kV}$, c) stressed at $10 \mathrm{kV}$, during volts off condition.

observation can be seen at all three stressing voltage and more noticeable as the voltage increases. On the other hand, charge density in the $\alpha$-methylstyrene soaked LDPE as illustrated in Figure 4, shows a different characteristic. As the voltage increases, more positive charge accumulation can be seen in the bulk of the sample and near the anode.

At $10 \mathrm{kV}$, positive charges dominate the bulk of the sample and the amount is rising as the sample is stressed for a longer time. Somehow, $\alpha$-methylstyrene assists the movement of the positive charges into the bulk. From the volts off results, we can conclude that both acetophenone and $\alpha$-methylstyrene enhance the movement of carriers in the polymer. However, the former seems in favour of the negative charge and the latter in favour of the positive charges.

\section{Space charge decay}

The influence of soaked chemicals on charge dynamics can also be studied by monitoring charge decay after the applied voltage is removed. Figure 5 shows the change in charge profiles in all three samples after the removal of the applied voltage. The clean LDPE shows a very slow charge decay rate as more charges are left in the bulk of sample even after 60 minutes. The negative charges near the top electrode shows an increment in density as more positive charges possibly leak 

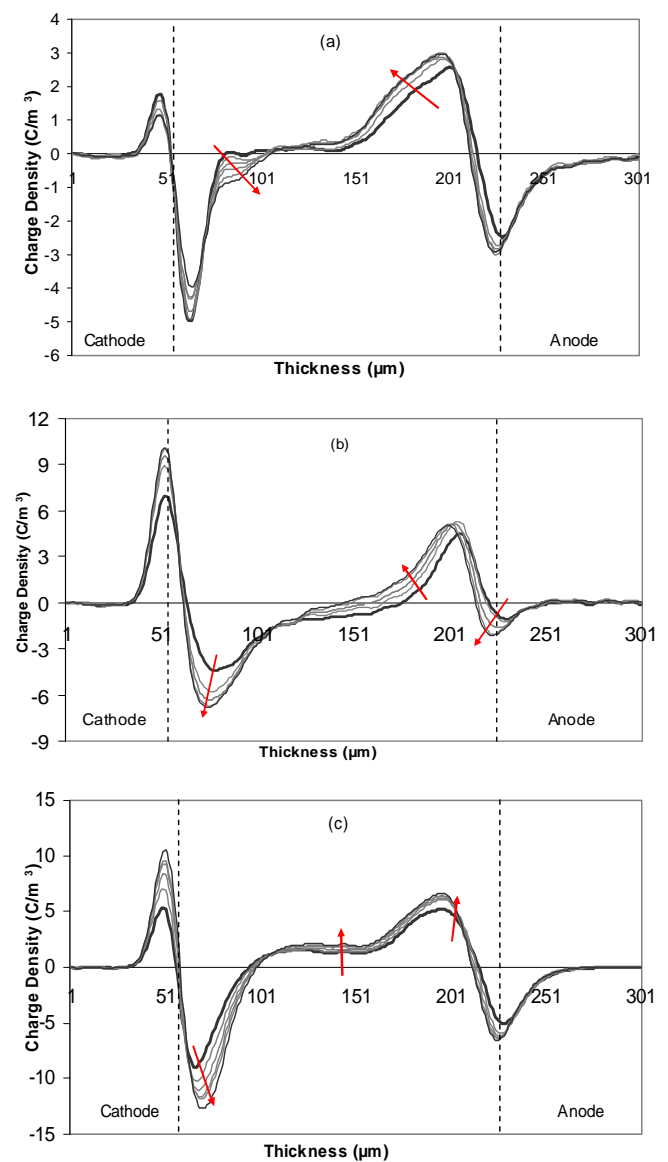

Figure 4: Charge dynamics in $\alpha$-methylstyrene soaked LDPE; a) stressed at $5 \mathrm{kV}, \mathrm{b}$ ) stressed at $8 \mathrm{kV}, \mathrm{c}$ ) stressed at $10 \mathrm{kV}$, during volts off condition.

into the anode, leaving the negative charges in the vicinity of electrode.

For acetophenone and $\alpha$-methylstyrene soaked samples, the decays are extremely fast especially in the first 10 minutes. The charges are believed to conduct into the electrode as the charge decay in the bulk is not as much as that near the electrodes. However, after 1 hour of decay, there are still some 'spots' that retain the initial amount of charges. It is believed that this observation may be due to the charges that are trapped in the deep traps that originally exist in the LDPE.

As the decay results are referred, acetophenone and $\alpha$ methylstyrene assist the movement of the carriers in LDPE and reduce the number of charges trapped in the existing traps of the polymer. This argument is supported by the results from volts off measurements, where more charges could drift into the bulk instead of trapped in the regions adjacent to the electrodes. Although these byproducts are in favour of different polarity of charges, the magnitude of accumulated charges is maintained or even lower than that seen in the clean LDPE.

The observation from space charge results are supported from the integration of the charge density in the sample bulk which gives the total charge. The total charge, positive charge and negative charge can be computed based on the charge profiles shown in Figure 2 to Figure 5. The effect of the
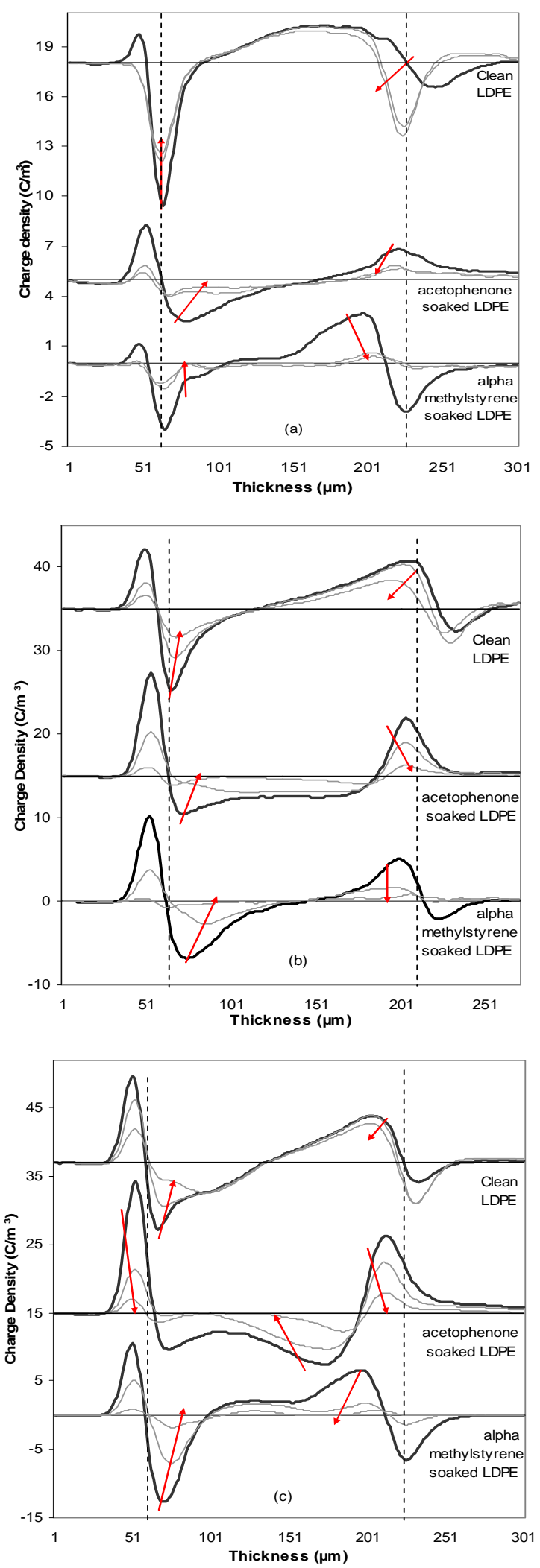

Figure 5: Charge decay after the removal of the applied voltage in three samples, a) stressed at $5 \mathrm{kV}$, b) stressed at $8 \mathrm{kV}$, c) stressed at $10 \mathrm{kV}$ for 1 hour.

chemicals on the total charge is utmost at elevated voltage and do not differ much at lower voltage. The result of the 


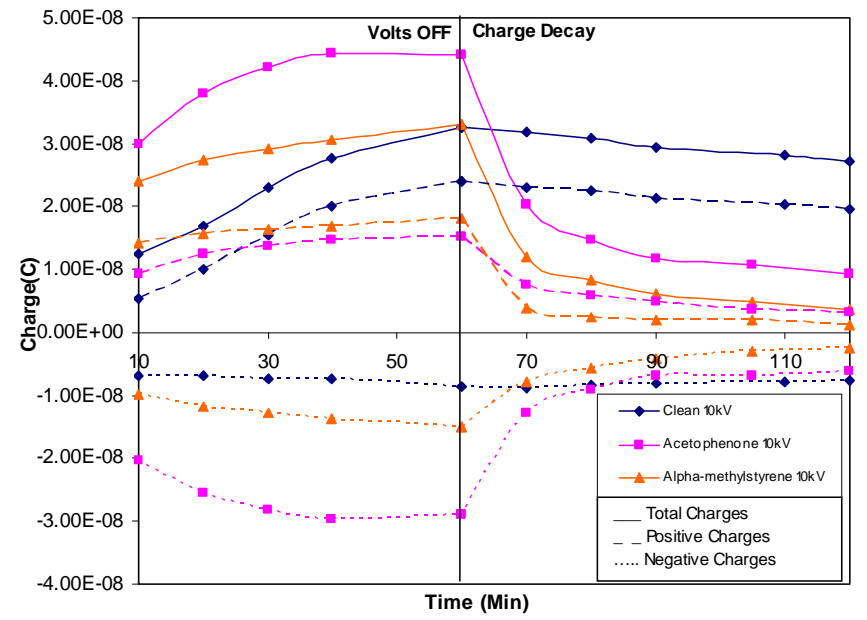

Figure 6: The total, positive and negative charges in the samples stressed at $10 \mathrm{kV}$.

calculation at $10 \mathrm{kV}$ is presented in Figure 6. Acetophenone soaked LDPE causes more charge injection, followed by $\alpha$ methylstyrene and then, clean LDPE. Acetophenone is more dominant in causing total charge injection compared to $\alpha$ methylstyrene. For negative charge injection, acetophenone gives a bigger impact. On the other hand, more positive charge injection can be observed in $\alpha$-methylstyrene soaked LDPE sample. In the first 10 minutes, the positive charges decay faster in $\alpha$-methylstyrene soaked LDPE samples meanwhile negative charges decay faster in acetophenone soaked LDPE samples.

There are two possibilities that could lead to this result. Firstly, acetophenone and $\alpha$-methylstyrene provide shallow traps in the soaked LDPE. When the LDPE is soaked with the chemicals, the small molecules may fill in the voids between the chains in the amorphous region. With only gas in the void in the clean LDPE, the charges travel towards the opposite electrode through hopping thermally activated conduction mechanism, reach the void surface and trapped upon the surface because of the negative electron affinity of PE $[6,7]$. When the chemicals fill into the void and they may form shallow traps, these traps will ease the movement of the charges over the space that used to be a void.

However, the above explanation is contrary to the theory and calculation using the Density Functional Theory (DFT) In this calculation the carbonyl group (acetophenone) or conjugate double bond ( $\alpha$-methylstyrene) will produce the deepest traps [8]. Using this model, the trap dept for acetophenone and $\alpha$-methylstyrene are $0.9 \mathrm{eV}$ and $1.53 \mathrm{eV}$ respectively [9]. This argument bring us to another possibility, which is acetophenone and $\alpha$-methylstyrene introduce various deep traps and thus, the charges tunnel between the traps and causes conduction. Rate of transfer between a pair of energy state of traps is given by [10];

$$
p=w \exp (-\alpha R) \exp (-W / k T)
$$

where $\mathrm{R}$ is the spacing between the states and $w, \alpha$ and $\mathrm{W}$ depend in a complicated way on the activation energy, the polarization energy and the degree of localization at each state. As the spacing between the states of traps is reduced as more deep traps are introduced, the rate of charge transfer will also increase. Nevertheless, there is no strong evidence to support this argument. In [11], the authors proposed an alternative mechanism of charge acceleration in acetophenone and $\alpha$-methylstyrene due to presence of the benzene ring and double bond in both chemicals' structure. However, further investigation is required before a firm understanding can be reached.

\section{CONCLUSION}

It is obvious that the presence of the byproducts changes the charge pattern in the samples. Acetophenone seems to assist the movement of negative charges into the bulk of sample while the $\alpha$-methylstyrene aids the transportation of positive charges. It seems that the chemicals may provide shallow traps in the insulation system that help charges to move faster. Further investigation is required to underpin the role of crosslinking byproducts in charge trapping and transportation.

\section{REFERENCES}

[1] T. Andrews, R. N. Hampton, A. Smedberg, D. Waldm, V. Waschk, W. Weissenberg, The Role of Degassing in XLPE Power Cable Manufacture IEEE Electrical Insulation Magazine 22(6): p. 5-16, December 2006

[2] W. A. Thue, Electrical Power Cable Engineering, $2^{\text {nd }}$ Edtion, New York, USA: Marcel Dekker, 2003.

[3]. Y. Ohki, N. Hirai, K. Kobayashi, R Minami, M. Okashita, and T. Maeno. Effects of byproducts of crosslinking agent on space charge formation in polyethylene -comparison between acetophenone and alpha-methystyrene. in Conference on Electrical Insulation and Dielectric Phenomena: IEEE, 2000 of Conference.

[4] N. Hirail, Y. Maeno', T. Tanaka, Y. Ohki', M. Okashita, and T. Maeno, Effect of Crosslinking on Space Charge Formation in Crosslinked Polyethylene. Properties and Applications of Dielectric Materials, June 1-5: p. 917-920. 2003.

[5] G. Chen, Y. L Chong and M Fu, Calibration of Pulse electroacoustic technique in the presence of trapped charge. Institute of Physicys Meas. Sci. Technol, 17: p. 1974-1980 2006.

[6] N. Ueno, K. Sugita, K. Seki and H. Inokuchi, Low-energy transmission and secondary-electron emission experiments on crystalline and molten long-chain alkanes. Phys. Rev., Volume 34(Issue 9): p. 6386 - 6393. 1986.

[7] G. Mazzanti , G. C. Montanari and S. Serra. Aging model of Polyethylene-based materials for $W$ cables founded on damage inception and growth from air-filled voids. in 2004 Inremotional Conference on Solid Dielectrics, Toulouse, Fronce,: IEEE, 2004 of Conference.

[8] M. Meunier, N. Quirke, A. Aslanides. Characterisation of Charge Carrier Traps in Polymeric Insulator in Conference on Electrical Insulation and Dielectric Phenomena, 2000.

[9] G. Teyssedre, C. Laurent Charge transport modeling in insulating polymers: from molecular to macroscopic scale IEEE Transactions on Dielectrics and Electrical Insulation, Vol 12(No 5): p. 857 - 875. October 2005.

[10] J. P. Jones, J. P. Llewellyn and T. J. Lewis, The Contribution of FieldInduced Morphological Change to the Electrical Aging and Breakdown of Polyethylene. IEEE Transactions on Dielectrics and Electrical Insulation, Vol 12 (No 5): p. 951-966. 2005.

[11] N. Hirai, R. Minami, Y. Ohki, M. Okashita and T. Maeno, Effect of Byproducts of Dicumyl Peroxide on space charge formation in Polyethylene in IEEE 7th International Conference on Solid Dielectrics, Eindhoven, the Netherlands: p.450 -455, 2001. 